\title{
A pilot-based grouping method in satellite-terrestrial cooperative wireless networks
}

\author{
Jhengsian $\mathrm{Li}^{1,2}$, Chunghua Chiang ${ }^{3}$ and Jyhhorng Wen ${ }^{4, ~ a ~}$ \\ ${ }^{1}$ Department of Communication Engineering, National Chung Cheng University, Chiayi, Taiwan, R.O.C \\ ${ }^{2}$ Chunghwa Telecom Laboratories, Taoyuan, Taiwan, R.O.C. \\ ${ }^{3}$ Department of Electronical Engineering, National Chung Cheng University, Chiayi, Taiwan, R.O.C. \\ ${ }^{4}$ Department of Electrical Engineering, Tunghai University, Taichung, Taiwan, R.O.C.
}

\begin{abstract}
In this paper we examine the performance of a satellite-terrestrial cooperative wireless network. We proposed a new grouping method, called pilot-based grouping method, to implement the space-time code in the satellite-terrestrial networks. The proposed method increases the total system channel capacity, and also saves unnecessary transmission power for the terrestrial stations. The proposed method focuses on finding out the best two signals coming from its neighboring terrestrial stations and uses the advantage of space diversity to improve the effect of channel fading. The numerical results show that the performance of ours proposed method is better than traditional grouping method.
\end{abstract}

Keywords: satellite-terrestrial networks; cooperative; space-time code.

\section{Introduction}

Satellite-terrestrial wireless network has become a complicated issue worth investigation in greater depth over the last few years. The satellite system can provide signals for large coverage in rural areas, but there are some limitations in the indoor environments because of signal blocking, especially in urban areas. The terrestrial networks, such as cellular networks, can provide good coverage in dense urban areas with a large number of base stations, but are not efficient for rural areas. The combination of the satellite-terrestrial network and cooperative network leads to the benefits from the aforementioned systems.

Multimedia broadcast and multicast service (MBMS) will play an important role in the future. Considering the power consumption and the antenna size of user equipment, unidirectional service is more suitable for satellite-terrestrial network. With its unidirectional nature, satellite-terrestrial can be a suitable technique to provide MBMS in both rural and urban areas.

The concept of transmit diversity requires multiple antennas at the transmitter. But in many communication systems, it is not practical to install multiple antennas. The idea of using other single antenna device as a relay to share their antenna and to generate a virtual multiple antenna system is called cooperative communication network [1-15]. In Satellite-Terrestrial Wireless Networks, the terrestrial node is treated as the relays to retransmit the signal [16-24]. In addition to providing better performance, the space-time coding in satellite terrestrial wireless was proposed in [1-2].

In Canada, XM Satellite Radio (XMSR) implements terrestrial repeaters in dense urban cities to

\footnotetext{
${ }^{\text {a } C o r r e s p o n d i n g ~ a u t h o r ~: ~ j h w e n @ t h u . e d u . t w ~}$
} 
boost the satellite signals for its digital radio service. Mobile Satellite Ventures (MSV) integrates the satellite and terrestrial cellular system to offer satellite telephony. This means the phones is compatible with both the upcoming MSV satphone network and local cellular networks. In Korea, Satellite Digital Multimedia Broadcasting (S-DMB) system has also started the commercial service since 2006 [25]. S-DMB also uses terrestrial repeater network for indoor coverage in urban areas.

In [2], the terrestrial stations in the satellite-terrestrial system are separated into several groups, and each group uses different frequency while the terrestrial stations in the same group are designed to use the same frequency. The user terminal performs space time code (STC) decoding process for each group. Finally, all the decoded signals in different frequencies are combined to recover the original signals. In this system, the transmitting power is inefficient because not all the relay stations are close enough to the user equipment. In other hand, the user equipment needs to receive from different groups in different frequencies. While the number of groups increases, the process time of user equipment also extends.

In this paper, a pilot-based terrestrial stations grouping method with less power consumption, faster user equipment processing time and higher total system channel capacity is proposed.

So, we propose a new grouping method of the terrestrial stations with less power consumption, faster user equipment processing time and higher total system channel capacity. In the following section, the system model and satellite-terrestrial network are discussed. In section 2.1, the traditional grouping method in terrestrial station is introduced. The proposed pilot-based grouping method is given in section 2.2. The numerical results of the proposed grouping method are compared with the previous research in section 2.3. Finally, the conclusions are presented in section 3.

\section{System model of the satellite-terrestrial network}

The are several different satellite multimedia broadcasting systems in the world, such as XM Satellite Radio and Mobile Satellite Ventures (MSV) in Canada, S-DMB in Korea, and etc. All of the systems need the terrestrial station to enhance the signal for the user in dense urban and buildings.

A basic mobile satellite broadcasting system is illustrated in figure 1. The contents, such as audio, video...etc., are sent to the satellite by the broadcast center and the satellite broadcasts the data to the terrestrial stations and user equipment. Then, the terrestrial stations transmits the received signals to the user. So, there will be two paths to the user terminal (i.e., the direct path from satellite and the path via terrestrial station).

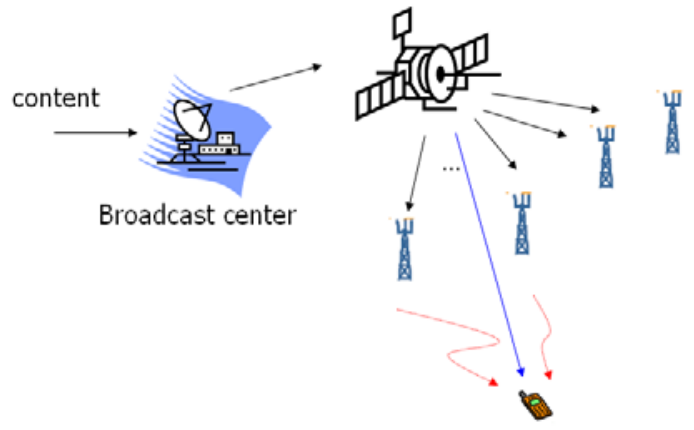

Figure 1. Basic structure of mobile satellite broadcasting system

This kind of structure is a unidirectional broadcasting system. The satellite-terrestrial system will be able to provide bidirectional communication if the user equipment can communicate with the terrestrial station. It is achievable if the existed cellular network and the satellite broadcasting center are integrated. The user equipment can send their request to the broadcasting center via the cellular network.

In Korea, the commercial S-DMB service has started in May 2005 [21]. In order to provide services to the areas shadowed or blocked by buildings, on-channel repeaters called gap fillers are 
used as the terrestrial stations. If the terrestrial station only plays the role as a repeater, the bandwidth usage will be the same as the satellite. For example, the total bandwidth of the S-DMB system in Korea is $25 \mathrm{MHz}$, and the system provides about maximum 30 channels. The repeater will also use $25 \mathrm{MHz}$ bandwidth to transmit in these 30 channels. Considering the multicast service or audio on demand service, the terrestrial station only needs to transmit part of the channels required by the users within its coverage. In the service on demand scenario, the number of usage content channels can be more than the actual number of channels transmitted simultaneously in one terrestrial station. The variety of the content service is a key factor to attract users in service on demand scenario. Also, the more channel capacity the terrestrial network can provide, the more subscribers can be served in the system.

\subsection{Traditional grouping method in terrestrial station}

In order to enhance the performance of the mobile satellite broadcasting system, the cooperative transmit diversity scheme is proposed in [1-2], and the figure 2 shows the proposed method in [2].

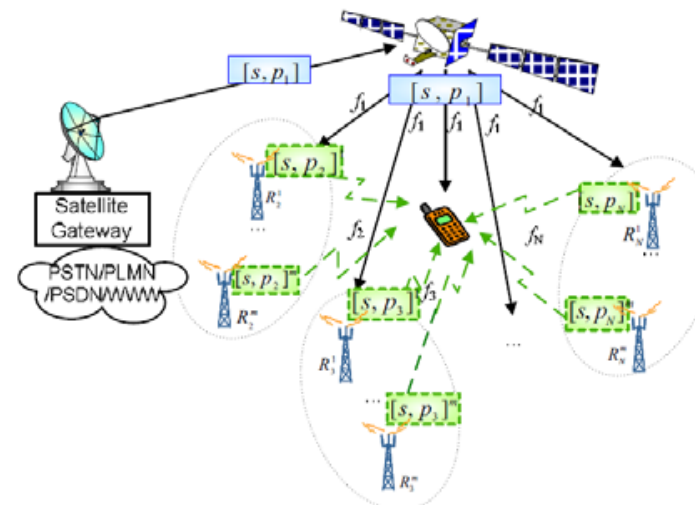

Figure 2. System model for cooperative satellite-terrestrial network in [2]

In [2], a rate compatible turbo coder generates an encoded signal sequence of $\left[s, P_{1}, P_{2}, \ldots, P_{N}\right]$ with code rate of $1 /(N+1)$ in the transmitter of the satellite gateway. In the encoded sequence, $s$ and $P_{i}$ $(1 \leq i \leq N)$ stand for a systematic and the $i$-th parity parts, respectively. The information is assumed to be decoded by the systematic part and any combination of the parity parts. The satellite transmits the sequence $\left[s, P_{i}\right]$ to the user terminal and all $\mathrm{m} \cdot(N-1)$ repeaters. When a repeater $R j i(2 \leq i \leq N)$ receives $\left[s, P_{i}\right]$, then it first generates another parity part $P_{i}$ by using the same encoder used in the gateway transmitter. Second it generates STC signal format, $\left[s, P_{i}\right]^{j}$ by using the sequence $\left[s, P_{i}\right]$. The repeater, now sends the encoded sequence $\left[s, P_{i}\right]^{j}$ to the user terminal using the frequency band of $f_{i}$.

If the user terminal receives multiple signals from the repeaters and also from the satellite, then it first performs STC decoding process using multiple signals with the same frequency band $f_{i}$ and recovers the sequence $\left[s, P_{i}\right]$. This STC decoding process is performed for all available frequency bands at the terminal. The next step is to combine $\left[s, P_{1}\right],\left[s, P_{2}\right], \ldots$, and $\left[s, P_{N}\right]$ to generate the mother code or a higher rate code, and apply the iterative decoding to recover the original information. Even though one of the paths might be missed, the information could still be recovered. This is because the rate compatible code used in the system is self-decodable.

Considering the grouping method in [2], named it as "all frequency allocated grouping method", the STC gain only happens in the case of receiving both signals coming from the STC pair stations of the same group. In this method, if there are $(N-1)$ frequencies available for the terrestrial stations, all the stations are divided into $(N-1)$ groups. Each group consists of two stations and both two stations transmit signals in the same frequency. Regarding the environment, the channel propagation path loss and fading between each station and the user will be taken into account in this paper. The propagation path loss $L_{i j}$ is defined as the power law of the distance between the $i$-th station and the $j$-th user. 


$$
L_{i j}=d_{i j}^{-\gamma}
$$

where $d_{i j}$ denotes the distance between $i$-th station and the $j$-th user, and $\gamma$ is the path loss exponent, which is equivalent to 2 in this paper. The fading, $X_{i j}$, is assumed to be an i.i.d. Rayleigh random variable with a unit variance. Combining the propagation path loss and the fading, the channel link gain $h_{i j}$ can be represented as (2).

$$
\left|h_{i j}\right|^{2}=d_{i j}^{-\gamma}\left|X_{i j}\right|^{2}
$$

when the user receives the STC signals coming from the same group, the expectation value of SNR after STC decoding can be shown as (3)

$$
E[S N R]=\frac{P_{r}}{N_{0}}\left(\left|h_{2 i-1}\right|^{2}+\left|h_{2 i}\right|^{2}\right)=\frac{P_{r}}{N_{0}}\left(d_{2 i-1}^{-\gamma}+d_{2 i}{ }^{-\gamma}\right)
$$

Maximum ratio combining (MRC) can be applied after receiving signals coming from each group. Then the expectation value of SNR after MRC can be expressed as (4)

$$
E[S N R]=\frac{P_{r}}{N_{0}}\left(\left|h_{1}\right|^{2}+\left|h_{2}\right|^{2}+\cdots+\left|h_{2(N-1)}\right|^{2}\right)=\frac{P_{r}}{N_{0}}\left(d_{1}^{-\gamma}+d_{2}^{-\gamma}+\cdots+d_{2(N-1)}^{-\gamma}\right)
$$

the channel capacity of a user can be shown as (5) [26],

$$
\begin{aligned}
& C=\frac{1}{N-1} \cdot E[\log (1+S N R)] \approx \frac{1}{N-1} \cdot \log (1+E[S N R]) \\
& =\frac{1}{N-1} \cdot \log \left[1+\frac{P_{r}}{N_{0}}\left(d_{1}^{-\gamma}+d_{2}^{-\gamma}+\cdots+d_{2(N-1)}{ }^{-\gamma}\right)\right]
\end{aligned}
$$

the coefficient $1 /(N-1)$ is a time factor that a user needed to decode the signals coming from $(N-1)$ groups/frequencies. If it is a broadcasting system, no matter how many users are in the system, the channel capacity can be described as (5). But if it is a service by demand system, different data types are sent to different users. When the number of user $m$ increases, as long as the $m \leq(N-1)$, the station in different groups takes turns to transmit data to different users simultaneously. But once $m>(N-1)$, it will not to transmit data from $(N-1)$ group to $m$ different users simultaneously. The channel capacity should be (6)

$$
C=\frac{1}{\left(1+\operatorname{floor}\left(\frac{m}{N-1}\right)\right) \cdot(N-1)} \cdot \log \left[1+\frac{P_{r}}{N_{0}}\left(d_{1}{ }^{-\gamma}+d_{2}^{-\gamma}+\cdots+d_{2(N-1)}{ }^{-\gamma}\right)\right]
$$

where the $(1+$ floor $(m /(N-1)))$ term represents that the system can serve the $N$-th user until the data of the previous $(N-1)$ user are sent. The more users in the system, the more waiting time will be needed for the system to serve each user. There are several problems that will degrade the performance of the system:

- The receiving time for the user equipment will be long while the number of the group/stations is large. For example, if there are $(N-1)$ groups, and $2 \mathrm{~T}$ stands for the two sub time slot. The time for user equipment to receive a signal coming from all the group will be $(N-1) \cdot 2 T$.

- The relays in different groups transmit signals in different frequencies, and the user equipment cannot receive all the frequency at the same time. So, the user equipment will need to synchronize and to each relay in proper time. It will be complicated.

- When a user located at the position between two groups, the user may only receive from only one group. In this situation, the STC gain does not exist even two different signals have been received by the user. 
- Not all the terrestrial station signals are able to be received. For those stations that are far away from the user, the transmitted power is wasted.

- For the case of multicast service or services on demand, it will take much longer time for users to wait while more users in the system.

\subsection{Pilot-based grouping method}

In order to solve the problems mentioned in section 2.1, we propose a new terrestrial station grouping method called "pilot-based grouping method" (shown in figure 3).

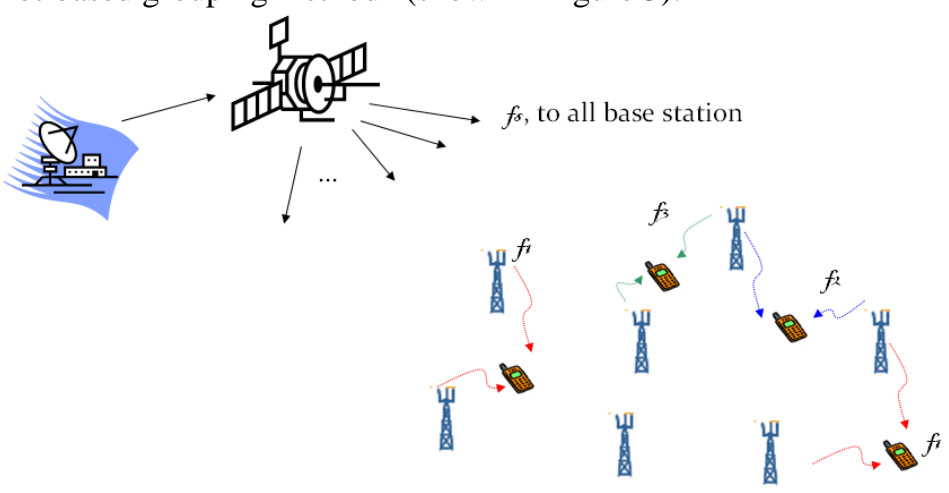

Figure 3. System model of pilot-based grouping method

In our proposed method, every terrestrial station is capable of transmitting signals in the whole available frequency band $\left(f_{1}, f_{2} \ldots, f_{N-1}\right)$, and the maximum transmission power is $P_{r}$ for each relay station (terrestrial station). Each user should be served by the best two relay stations to form a STC pair. Every station will transmit a predefined pilot signal in the center frequency to identify itself. The user in the network will detect the pilot channel and report the best two pilot signals to the system. The pilot channel link gains may not be the same in a frequency selected fading environment. Since the key point is to find out the best two among all the relays, using central frequency as an indication of expected channel link gain comparison results should be reasonable to represent the comparison results of all the other frequencies. The best relay will allocate an available frequency to the user, and the second best relay will follow the same frequency chosen by the best relay in order to form a STC pair. The pilot channel assignment is illustrated in figure 4 .

Three terrestrial stations transmit their own pilot channel in the same center frequency. User 1 is served by a pair of relay stations using blue spectrum. Although User 2 measures the best pilot signal coming from station $\mathrm{C}$ and second best coming from station $\mathrm{B}$, the same frequency used by user 1 cannot allocate to user 2 .
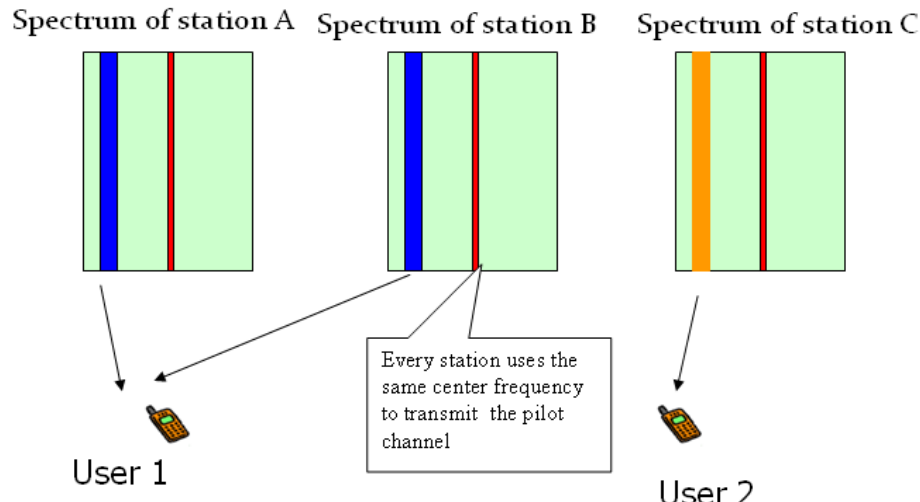

Figure 4. Pilot channel in the system and frequency assigning 
The basic concept of the pilot-based grouping method is that every user should be served by the best two relays to offer STC, and the same frequency can't be allocated to different users at the same time. The user will receive the STC signals of the best two relays, and the expected value of SNR can be represented as (7). The channel capacity is given in (8).

$$
\begin{gathered}
E[S N R]=\frac{P_{r}}{N-1} \cdot \frac{1}{N_{0}}\left(\left|h_{1}\right|^{2}+\left|h_{2}\right|^{2}\right)=\frac{P_{r}}{(N-1) \cdot N_{0}}\left(d_{1}^{-\gamma}+d_{2}^{-\gamma}\right) \\
C=E[\log (1+S N R)] \approx \log (1+E[S N R])=\log \left[1+\frac{P_{r}}{(N-1) \cdot N_{0}}\left(d_{1}^{-\gamma}+d_{2}^{-\gamma}\right)\right]
\end{gathered}
$$

The steps of the pilot-based grouping method include initialization and relay grouping listed as follow:

Initialization:

1. Let number of total relay $N=2^{2 n}(n=1,2,3, \ldots)$, and number of available frequency $N / 2$. The relays are uniformly distributed in a square region (length $\cdot$ width $=2^{n} \cdot 2 n$ at positions $(i+0.5, j+$ $0.5)$, where $i, j=0,1,2, \ldots, 2^{n-1}$ )

2. Set each relay total power $\left(P_{r}\right)$

3. All the available frequencies are free to use in every relay

4. The total number of user $=M$

5 . Set the total grouping success counter, succ_t , to 0 . Set the total grouping fail counter, fail_t $t$, to 0 .

Set the total channel capacity, capa_t, to 0

Relay grouping:

6 . Assume the users arrive randomly in the system one by one. Then, the relay grouping successful rate and channel capacity of the $m$-th user will be calculated. ( $m=0$ as initial value)

7. $m=m+1$. Measure the all the pilot coming from all the $N$ relays. Choose the best and second strong pilot channel named and then named these two relays as Best_R_m and Second $R \_m$

8. If the frequency of Best_R_m is unavailable, then fail_t $t=$ fail_t $t+1$.

9. Else, randomly choose one of the available frequencies $f_{m}$ in Best_R_m for user $m$.

10. If the same frequency $f_{m}$ in Second $R \_m$ is available, allocate $f_{m}$ in both Best_R $R$ and Second_R_m to user $m$. Then $s u c c \_t=s u c c \_t+1$. Calculate the channel capacity, capa_m, of user $m$; then, $c a p a \_t=c a p a \_t+c a p a \_m$.

11. Else fail_t $=$ fail_t +1 ..

12. If $m<M$, go to step (7)

13. Calculate grouping_succ_rate $=$ succ_t $/\left(\operatorname{succ} \_t+\right.$ fail_t $\left.t\right)$.

14. End

\subsection{Numerical results}

A simple numerical is shown in Fig 5. The result shows that the pilot-based grouping method has more channel capacity. This result can be explained due to the $1 /(N-1)$ factor in the two methods. In all frequency allocated grouping method, the $1 /(N-1)$ factor degrades the channel capacity more severely than the pilot-based grouping method. When the number of users is less than $1 /(N-1)$, it is possible to arrange the relay stations transmitting different data to different user at the same time. But once the number of active users exceeded 1/ $(N-1)$,the system will need to arrange the data to be transmit in more than one time frame. So the coefficient should be $1 /((1+\operatorname{floor}(\mathrm{m} /(N-1)) \cdot(N-1)))$. This lead to the result of more total waiting time needed for each user to receive their data. 


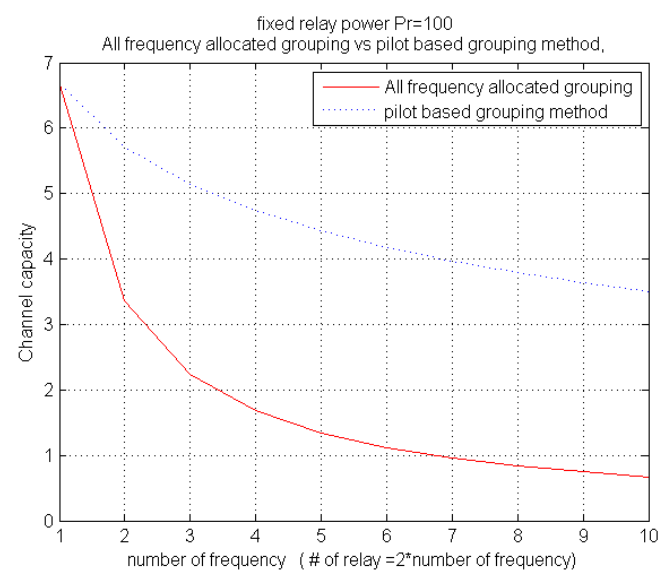

Figure 5. Simple comparison of two grouping methods

In order to compare with the algorithm proposed in [2], we assume that the number of terrestrial station is half of the available frequencies. The frequencies are assumed to be narrow band. The maximum power of each terrestrial relay station is the $P_{r}$. The relay stations located in a two dimension square area with fixed separation in $x$ and $y$ axis. In our numerical, the separation of one station to the neighboring station are one nuit both in $x$ and $y$ axis (see figure 6). The locations of the users are randomly generated.

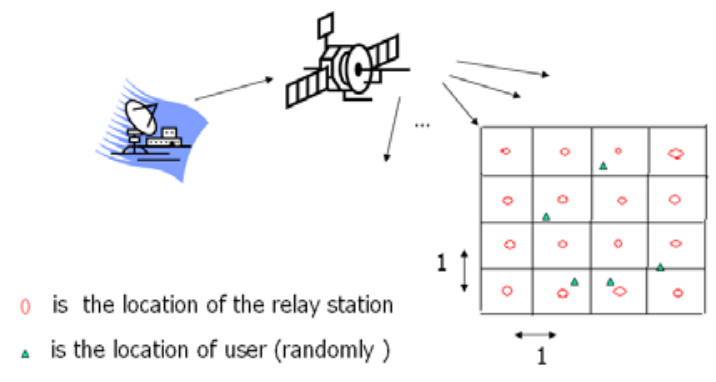

Figure 6. The locations of terrestrial station and users

Figure 7 shows the comparison result between "all frequency allocated grouping method" and "pilot based grouping method". Obviously, the pilot based grouping method has higher total channel capacity.

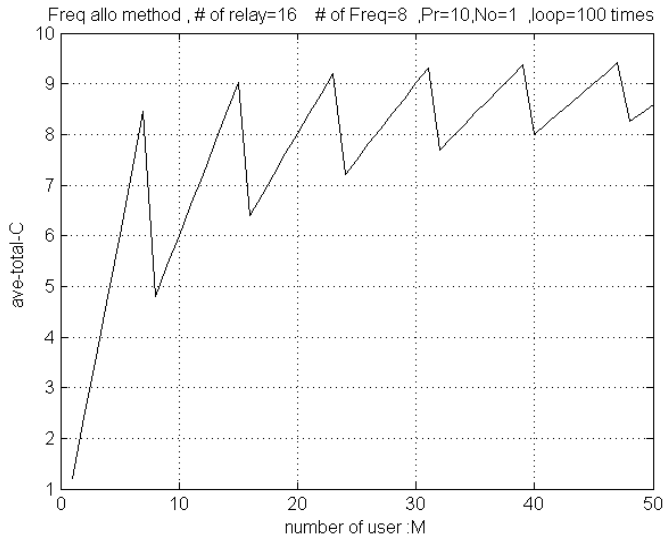

(a)All frequency allocated grouping method

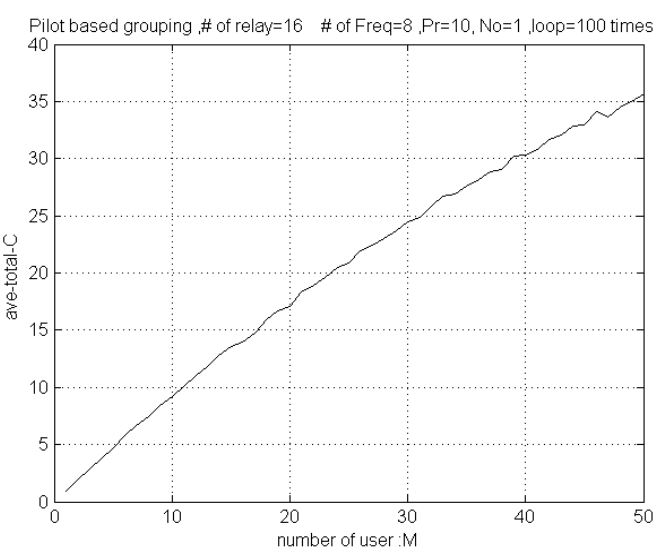

(b) Pilot-based grouping method

Figure 7. Total channel capacity comparison 


\section{Conclusions}

The combination of satellite and terrestrial network can provide good coverage in both rural and urban areas. The Pilot-based grouping method is proposed to increase the total system capacity, which facilitates the implementation of the service-on-demand satellite-terrestrial wireless network with various contents. For the pilot-based grouping methods, the user equipment only receives signals coming from the best two relays, and need not to switch frequencies to receive data from different relays. On the other hand, the pilot-based grouping methods can save power and bandwidth for the terrestrial stations far away from the user. The proposed method can find the best two relays to group together, and have better performance than all frequency allocated grouping method by space divinity.

\section{Acknowledgment}

This work was supported by National Science Council Grant (NSC 101-2221-E-029-020 -MY3)

\section{References}

1. S. Kim, H. W. Kim, K. Kang and D. S. Ahn, IEEE Commun. Mag., 46, 118 (2008)

2. S. Kim, H. Kim and D. S. Ahn, ASMS, 72 (2008)

3. K. J. R. Liu, A. K. Sadek, W. F. Su, and A. Keasinski, Cooperative Communications and Networking (Cambridge University Press, 2009)

4. Y. W. Hong, W. J. Huang, F. H. Chiu and C. C. J. Kuo, IEEE Signal Process. Mag., 24, 47 (2007)

5. A. Nosratinia,T. E. Hunter and A. Hedayat, IEEE Commun. Mag., 42, 74 (2004)

6. X. F. Tao, X. D. Xu, and Q. M. Cui, IEEE Commun. Mag., 50, 65 (2012)

7. W. Swasdio,C. Pirak, G. Ascheid, ICUMT, 1 (2011)

8. C. Esli and A. Wittneben, GLOBECOM, 3926 (2007)

9. Q. Q. Zheng, S. Kota, V. Lau, and W. F. Su, IEEE J. Sel. Topics Signal Process., 5, 1 (2011)

10. Y. Z. Yao and X. D. Dong, IEEE Trans. Veh. Technol., 62, 2865 (2013)

11. O. Amin and L. Lampe, IEEE Wireless Commun. Letters, 1, 412 (2012)

12. Z. Sun, I. F. Akyildiz, and G. P. Hancke, IEEE Trans. Wireless Commun., 10, 3793 (2011)

13. J. N. Laneman, D. N. C. Tse, Wornell, W. Gregory, IEEE Trans. Inf. Theory, 50, 3062 (2004)

14. C. Zhang, H. Yin, W. Wang and G. Wei, ChinaCom, 498 (2008)

15. X. Wu and L. L. Xie, IEEE Trans. Inf. Theory, 59, 2613 (2013)

16. J. Cai, X. Shen, J. W. Mark, A. S. Alfa, IEEE Trans. Wireless Commun., 7, 1348 (2008)

17. C. L. Wang and S. J. Syue, WCNC, 1 (2009)

18. S. Alamouti, IEEE J. Sel. Areas Commun., 16, 1451 (1998)

19. G. Singh, ICCCA, 1335 (2015)

20. Y. Celik, ICT, 1 (2016)

21. H. Yang, J. Zhang, Y. Ji, Y. He, and Y. Lee, Scientific Reports, 6, 30678 (2016)

22. H. Yang, J. Zhang, Y. Zhao, Y. Ji, J. Han, Y. Lin, and Y. Lee, IEEE Commun. Mag., 53, 130 (2015)

23. C. Yuan, M. Lin, O. Jian, and Y. Bu, IEEE VTC Spring, 1 (2016)

24. H. Yang, J. Zhang, Y. Zhao, J. Han, Y. Lin, and Y. Lee, IEEE Commun. Mag., 54, 86 (2016)

25. S. J. Lee, S. Lee, K. W. Kim and J. S. Seo, IEEE Trans. Broadcast., 53, 179 (2007)

26. T. M. Cover, and A. A. E. Gamal, IEEE Trans. Inf. Theory, 25, 572 (1979) 